\title{
APS Award to Professor Dr. H. Welker
}

W. Heywang, Munich

At the 1975 Spring Convention of the American Physical Society in Denver, a European, Professor Dr. Heinrich Welker became the first recipient of an award for research on new materials. In the tribute paid to Professor Welker some 25 years after his original discovery the award was substantiated as follows :

"For his pioneering investigations on the physical and chemical properties of the binary compounds of the elements in groups III and V of the Periodic Table. His programme of research and development, carried out with the explicit intention of enlarging the family of useful semiconductors, has made many new devices and techniques possible".

Professor Welker's discovery resulted from a search for new semiconductor materials which was prompted by his early work in developing highpurity germanium for microwave detectors. Fascinated by the extremely small effective mass of the conduction electrons, which was exceptional among the then known semiconductors, he was curious as to how these would react to practical substitutions. Guided by the work of Sommerfeld and Grimm on the theory of compounds, he deduced that the III-V compounds which crystallize in the closely allied zincblende crystal structure, where quadrivalent germanium atoms are alternately replaced by trivalent and pentavalent atoms, must have interesting semiconducting properties (cf. Fig. 1).

In the Spring of 1951 he decided to test the tenability of this assumption by an experiment. Lacking more elaborate experimental facilities, he fused a rod of InSb in the cellar of his own house and thereby observed a remarkably strong Hall effect.

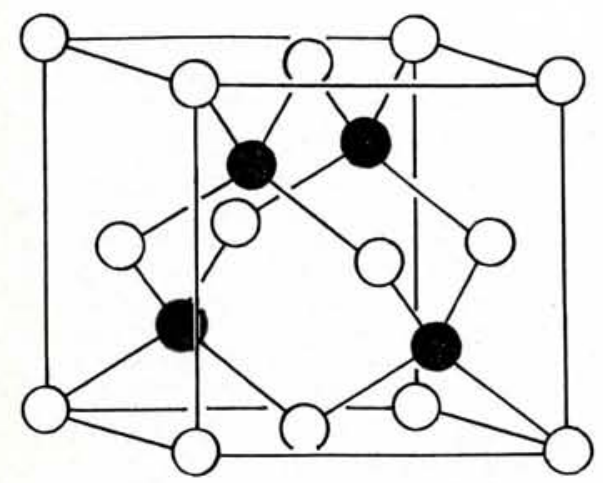

Fig. 1 Zincblende structure: the open circles represent a 3-valent ion (e. g. Ga) and the black circles a 5-valent ion (e. g. As)

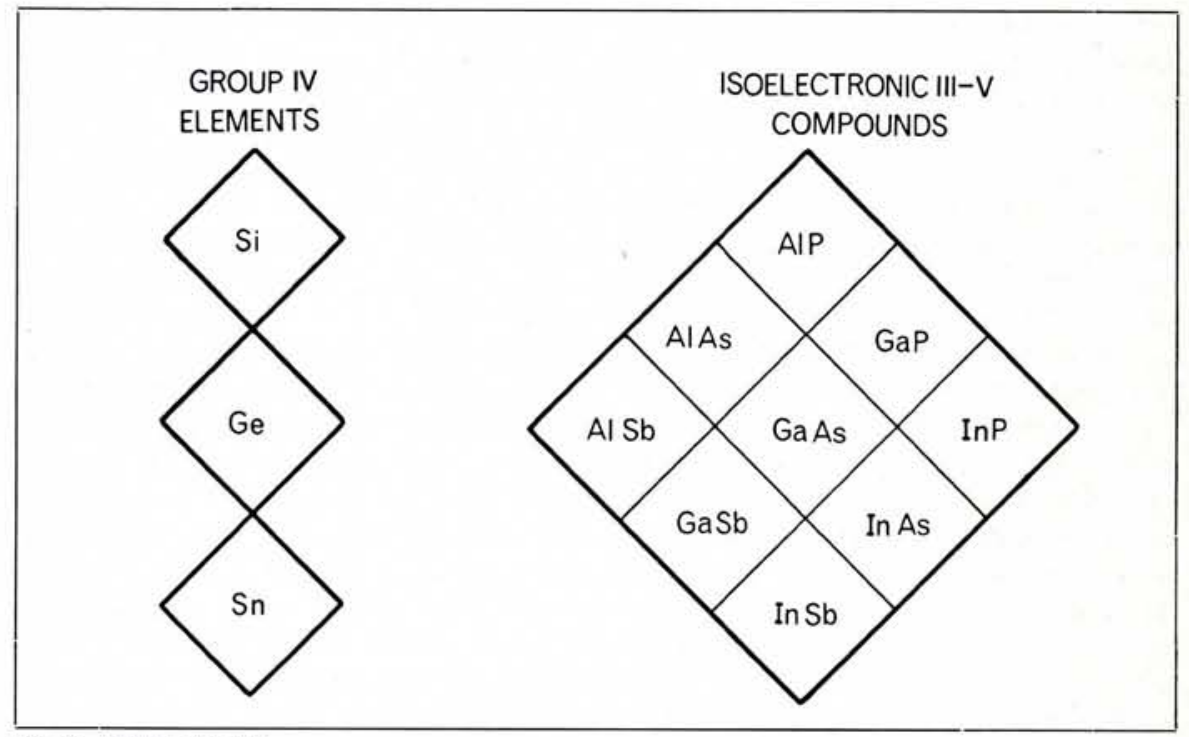

Fig. 2 Welker diagram

This event, which may be considered to mark the actual birth of the III-V semiconductor, also provided the basis which enabled him, on joining Siemens during the Summer of 1951 , to form a research group of his own for the investigation of semiconducting compounds. His first project was to synthesize the various possible compounds (cf. the diagram given by Welker in Figure 2) with the degree of purity - if possible in the monocrystalline form - required for semiconductor devices and to determine their properties. His prediction that all were semiconducting compounds proved to be true and it was found possible to determine systematic relations between the fusing points, band gaps and electron mobilities.

This was of particular interest because electron mobilities were sometimes found which by far exceeded all previous records (e.g. $10^{5} \mathrm{~cm}^{2} / \mathrm{Vsec}$ in InSb as compared to $3000 \mathrm{~cm}^{2} / \mathrm{Vsec}$ in germanium, which was in those days the primary semiconductor of interest).

The publication of this discovery naturally occupied the entire scientific world and was followed by the appearance of countless papers on the properties of III-V compounds in all parts of the globe. To obtain a complete understanding of the mechanisms of these properties it was first essential to gain a more intimate knowledge of the band structure of diamond semiconductors ( $\mathrm{Si}, \mathrm{Ge})$ and zincblende semiconductors (III-V, II-VI semiconductors).

We now come to a topic that runs right through the history of III-V semiconductors; the rivalry between the elemental semiconductors $\mathrm{Ge}$ and $\mathrm{Si}$ on one hand and the III-V semiconducting compounds on the other. Welker too came upon these new compounds during his efforts to determine whether there are allied substances which are in one or another respect superior in usefulness to germanium. Taking germanium, which by 1951 had been virtually developed to maturity, as a starting point, it was principally the band gap of only $0.7 \mathrm{eV}$ which disallowed its use at relatively high temperatures. An improvement appeared to be offered by not only III-V compounds but also silicon, which has already conquered and is still conquering an increasingly large share of the market. Does this necessarily imply that silicon is ousting III-V compounds? Hardly, for in reality developments are often complementary. Whereas the elemental semiconductor silicon has - if one compares substances with a comparable band gap - a far simpler technology, it is the III-V compounds which offer the greater variety of new semiconductor properties.

An indispensable aid in obtaining a clear idea of the technical limits and potentialities of the rival substances, germanium and silicon, were the adequate energy band diagrams determined by the pseudopotential method in the late 1950s and early 1960s. The III-V compounds and the wealth of relevant data on their properties here provided valuable reference material. The principal parameters responsible 
for the semiconductor properties of these compounds are shown in the energy pulse ( $\hbar k$ ) diagram (Fig. 3). Although the valence band is divided into several subbands it is still possible to operate in the first approximation with a centrally symmetric parabolic band. In the conduction band, however, secondary minima appear at or near the boundary of the Brillouin zone in the $/ 100 /$ or $/ 111 /$ direction in addition to the central minimum. Completely different semiconductor properties result, depending on the energy level of these minima. If the central minimum has the lowest energy level, extremely high electron mobility will be observed. Electrons brought into this minimum by external excitation will cross directly from the conduction to the valence band (cf. Fig. 3, (a)) and light emission will occur. If the secondary minima have the lowest level, the electron mobility will be lower. Electrons may only cross indirectly from these minima to the valence band (cf. Fig. 3, (b)) and long carrier lifetimes will be possible. Finally it should be added that if charge carriers of high mobility are forced by an applied electric field from the central minimum to a satellite minimum (cf. Fig. 3, (c)), negative resistances will appear (Gunn effect) which, for instance, have assumed importance for $\mathrm{GaAs}$ or InP microwave generators. Except for this Gunn effect the properties of III-V compounds which are of significance as compared to those of germanium and silicon were already experimentally demonstrated by Welker and coworkers and the resulting possibilities with regard to the large range of variation of the band gap recognized. At first, however, only galvanomagnetic devices, which utilize the high mobility of the majority carriers, made any headway.

The technology for more sensitive effects still had to be developed. It was here that past experience with silicon, especially in connection with the

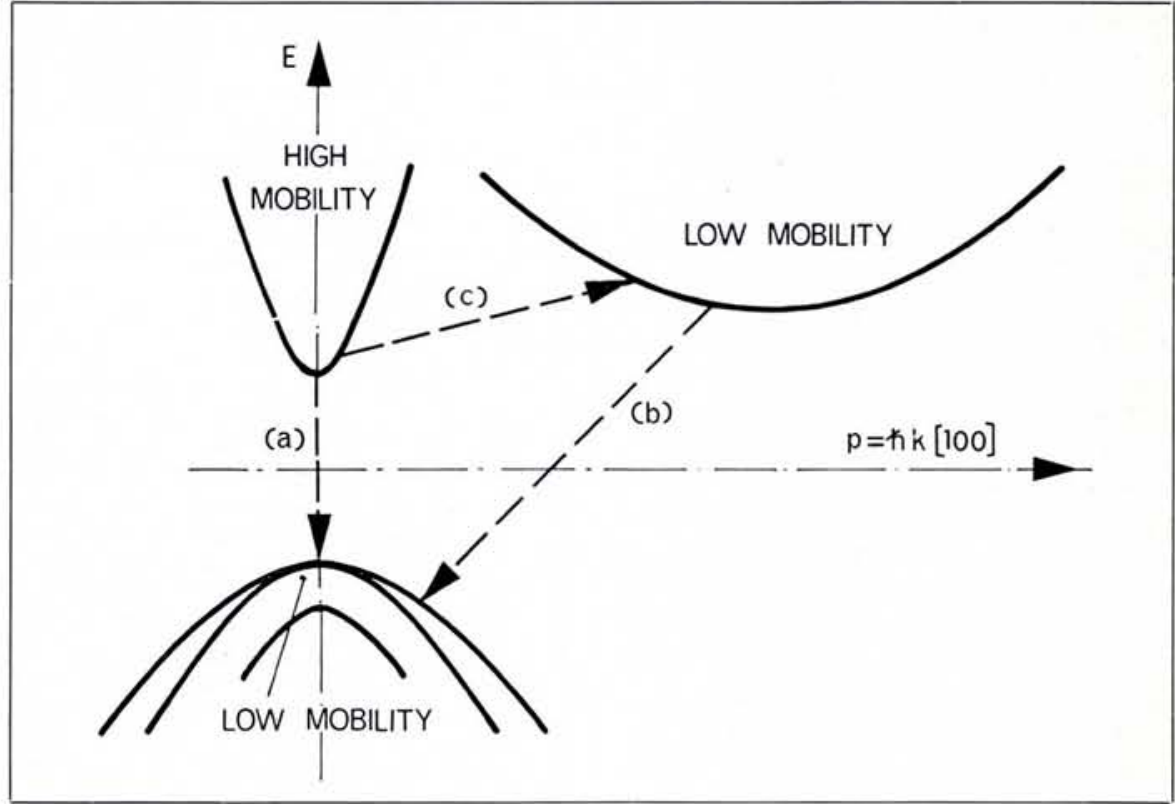

Fig. 3 Band structure diagram

deposition of ultrapure and perfect epitaxial layers, proved useful. Yet another impetus from the silicon sector proved of decisive importance for optoelectronics, the currently largest branch of III-V semiconductor technology: a demand for optoelectronic devices capable of operating with the same low voltages as integrated circuits. Thus the advent of GaAs, Ga (AsP) and $\mathrm{GaP}$ light-emitting diodes led to the creation of a market whose worldwide sales have already surpassed the $\$ 200$ Mill. mark. Not only would the simple LED with its broad spectrum extending from near infrared to light green be inconceivable without III-V compounds, but even the far more complex laser diodes built of GaAs and (GaAl)As layers could never have been realized without them. Designed to emit and amplify finely focused coherent light signals, this laser diode is ideally suitable for communications transmission. Like GaAs LEDs with their incoherent light it represents, by allowing easy modulation up to about the $\mathrm{GHz}$ region, a key device for future communication systems in which guided light waves will be transmitted along glass fibres. The future market can of course only begin to develop after the overall system has been brought to maturity.

Like that for the laser diode, the market for the large variety of microwave devices made of III-V compounds, GaAs Schottky-barrier diodes, GaAs tunnel diodes, GaAs FETs as well as GaAs and InP Gunn-effect elements is still in its infancy. These devices will only attract real interest after a sufficient demand has developed for frequencies in the region, of $10 \mathrm{GHz}$ and higher, where silicon approaches a limit set by its lower electron mobility.

Although this short review could only treat the principal aspects of III-V semiconductors, these alone already demonstrate what a vast and far-reaching area of science and technology has been opened up. Its importance has been adequately highlighted by the honorable award to the man who made this possible.

\section{3rd General Assembly of EPS Members}

The Third General Assembly of EPS members took place in Bucharest on Wednesday evening 10 September 1975. The President, H.B.G. Casimir, presented his report on the state of the society, stressing the difficult period passed by the Society due to the weak financial situation. He mentioned the personnel changes and further measures which have taken place at the Secretariat since Wiesbaden and he considered that the Society's acti- vities should be able to make further progress, backed by an organization that is in a healthy state. The President then gave a review of the most recent developments. Europhysics News was now being published according to recommendations made by an Advisory Committee and a new Advisory Committee on Physics Education was to have its constitutional meeting the following day.

The Secretary, M. Guenin, gave some details on administrative matters. The main problem was to find a way to improve the financial situation. Of the two possibilities, reducing expenditure or increasing the income, the first alternative had been applied to such an extent that new cuts would mean reducing the staff at the Secretariat to little more than zero. The Secretary announced the new publication Europhysics Conference Abstracts. He expressed the hope that 